\title{
Anisotropy Coupling in Patterned Thin Films with Mixed Uniaxial Anisotropies
}

\author{
Yoon Jae Nam ${ }^{1, a}$, Tae Young Lee ${ }^{1, a}$, and Sang Ho Lim ${ }^{1,2 *}$ \\ ${ }^{1}$ Department of Nano Semiconductor Engineering, Korea University, Seoul 136-713, Korea \\ ${ }^{2}$ Department of Materials Science and Engineering, Korea University, Seoul 136-713, Korea
}

(Received 7 May 2014, Received in final form 25 June 2014, Accepted 25 June 2014)

\begin{abstract}
Anisotropy coupling in thin films with mixed induced and shape anisotropies is investigated. A 200-nm-thick Co-Fe-Pd-B thin film with a large induced anisotropy of 57 Oe is fabricated and then patterned into micronsized cells to provide shape anisotropy, whose strength has a similar magnitude to that of the induced anisotropy for enhancing the anisotropy coupling. The angles between the two mixed anisotropies considered are $0^{\circ}, \mathbf{9 0}^{\circ}$, and $110^{\circ}$. Hysteresis loops measured under in-plane magnetic fields along various directions indicate no anisotropy coupling behaviour for all the three angles examined in this study.
\end{abstract}

Keywords : anisotropy coupling, mixed anisotropies, Co-Fe-Pd-B thin film, patterned magnetic cells

\section{Introduction}

Permanent magnets such as $\mathrm{Nd}_{2} \mathrm{Fe}_{14} \mathrm{~B}$ and $\mathrm{SmCo}_{5}$ exhibit very large coercivities (of the order of kOe), whereas soft magnetic materials such as permalloys and Si steels exhibit extremely small coercivities (of the order of mOe) [1]. This large difference in the coercivity is mainly due to a similarly large difference in the magnetic anisotropies of the two types of materials, thereby clearly demonstrating the dominant role of magnetic anisotropy in determining the magnetic properties. This magnetic anisotropy is magnetocrystalline anisotropy (MCA), which originates from intrinsic spin-orbit coupling [1]. In addition to this, there are many other types of anisotropies, including induced, shape, and magnetoelastic anisotropies. These anisotropies are often superimposed with MCA, with resulting changes in the magnetic properties. In the case of thin films fabricated by sputtering, for example, mostly unwanted residual stresses often occur due to the difference in the thermal expansion coefficients between the thin film and substrate. These stresses are combined with magnetostriction to form magnetoelastic anisotropy. Anisotropy is often induced deliberately to suit particular applications. Common examples include the induced aniso-

(C)The Korean Magnetics Society. All rights reserved.

aPresent address: SK hynix, Bubal-eup, Icheon-si, Gyeonggi-do 467-701, Korea.

*Corresponding author: Tel: +82-2-3290-3285

Fax: +82-2-928-3584, e-mail: sangholim@korea.ac.kr tropies formed by field-sputtering [2], field-annealing [3], and mechanical deformation [4]. It is therefore important to examine the change in magnetic properties as additional anisotropies are superimposed onto existing anisotropies.

To this end, Wohlfarth and Tonge examined the cases in which two uniaxial anisotropies are mixed together inside a magnetic system [5]. They proposed the following equation for the total energy $(E)$ for systems with two uniaxial anisotropies [5]:

$$
\begin{aligned}
E= & K_{u 1} \sin ^{2} \theta+K_{u 2} \sin ^{2}(\beta-\theta)+ \\
& \eta \sqrt{K_{u 1} K_{u 2}} \sin ^{2} \theta \sin ^{2}(\beta-\theta) \sin ^{2} \beta \\
& +M H \cos (\alpha-\theta)
\end{aligned}
$$

Here, $K_{\mathrm{u} 1}$ and $K_{\mathrm{u} 2}$ are the strengths of the two uniaxial anisotropies, $M$ is the magnetization, and $H$ is the applied magnetic field. The angles $\alpha, \beta$, and $\theta$ were defined with respect to $K_{\mathrm{u} 1}$, where $\alpha$ is the angle between $K_{\mathrm{u} 1}$ and $H, \beta$ is the angle between $K_{\mathrm{u} 1}$ and $K_{\mathrm{u} 2}$, and $\theta$ is the angle between $K_{\mathrm{u} 1}$ and $M$. The first two terms in Eq. (1) are due to the respective uniaxial anisotropies, whereas the last term results from the Zeeman energy. The third term, which contains both $K_{\mathrm{u} 1}$ and $K_{\mathrm{u} 2}$, represents the coupling of the two uniaxial anisotropies with the resultant anisotropy strength and direction determined by the coupling coefficient $\eta$, which ranges from 0 (no coupling) to 1 (full coupling). Figure 1 summarizes the coupling results predicted by Eq. (1) when two uniaxial anisotropies with different strengths $\left(K_{\mathrm{u} 1}>K_{\mathrm{u} 2}\right)$ are coupled together. Dis- 


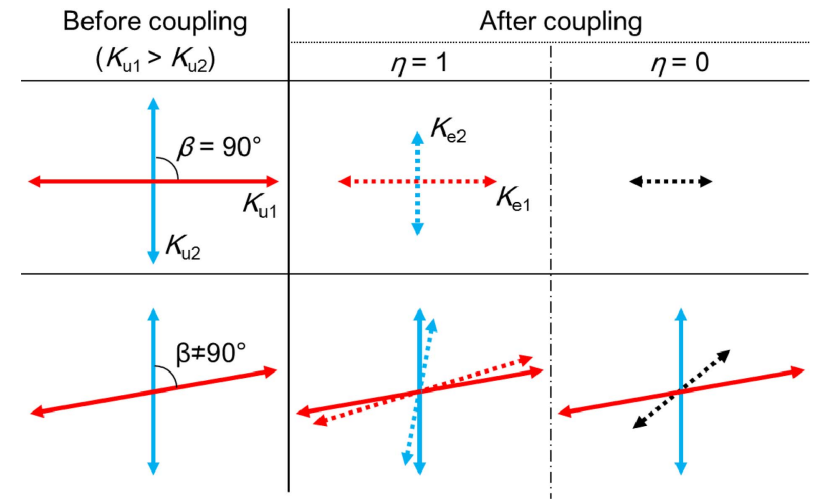

Fig. 1. (Color online) Summary of coupling results predicted by Eq. (1) when two uniaxial anisotropies with different strengths $\left(K_{\mathrm{u} 1}>K_{\mathrm{u} 2}\right)$ are coupled fully $(\eta=1)$ or not coupled at all $(\eta=0)$ for $\beta=90^{\circ}$ and $\beta \neq 90^{\circ}$.

played in the figure are two extreme cases of anisotropy coupling, $\eta=0$ and 1 . When two anisotropies cross at right angles $\left(\beta=90^{\circ}\right)$, the two anisotropies survive after coupling at $\eta=1$, but their strengths, indicated by $K_{\mathrm{e} 1}$ and $K_{\mathrm{e} 2}$, are reduced. At $\eta=0$, however, only the anisotropy with the greater strength survives, and the strength of the resultant anisotropy is obtained simply by the difference between the two anisotropy strengths. The situation is quite different when the crossing angle is not a right angle $\left(\beta \neq 90^{\circ}\right)$. For this case, both the direction and strength of the resultant anisotropies after coupling are different from those of the original anisotropy. The change in the anisotropy directions depends upon $\eta$. At $\eta=1$, two anisotropies persist even after coupling, but the crossing angle between the two anisotropies is reduced. Conversely, at $\eta$ $=0$, only one effective anisotropy exists, which is similar to the case of $\beta=90^{\circ}$. The resultant anisotropy is located at an intermediate angle between the two original anisotropies with less strength than each of the original anisotropies. Calculations using Eq. (1) for $\eta=0$ show the strongest destructive coupling (the weakest anisotropic strength after coupling) at $\beta=90^{\circ}$. The strength in the destructive coupling decreases as the angle deviates from the right angle.

Wohlfarth and Tonge predicted that $\eta=0$ if two mixed anisotropies are shape anisotropies, and $\eta=1$ if they are MCAs [5]. An intermediate value of $\eta$ may be obtained if MCA and shape anisotropy are mixed together [5]. Experimental studies then followed their theoretical predictions, using various systems with mixed anisotropies, consisting of MCA and shape anisotropies [6,7], and of magnetoelastic and shape anisotropies [8,9]. However, most of the previous studies were not conducted on systems with two uniaxial anisotropies, thereby making it difficult to test the model of Wohlfarth and Tonge. In a recent study using permalloy thin films with mixed shape and induced anisotropies [10], destructive interference was observed when the two anisotropies crossed at right angles. However, considering that the thin films studied therein have large dimensions (on the order of $\mathrm{mm}$ ) and the strength of the induced anisotropy is very small (4 Oe), the observed coupling behaviour is cast into doubt. For example, it is quite probable for the weak induced anisotropy to interfere with other parameters such as residual stresses [11] and misfit between the magnetic and buffer layers [12]. In this study, a Co-Fe-Pd-B thin film with a large induced anisotropy of 57 Oe was fabricated and the thin film was patterned into micron-sized cells to provide shape anisotropy, whose magnitude of strength is similar to that of the induced anisotropy.

\section{Experimental Details}

A 200-nm-thick amorphous Co-Fe-Pd-B thin film with a composition of $\mathrm{Co}_{41} \mathrm{Fe}_{16} \mathrm{Pd}_{28} \mathrm{~B}_{15}$ (at.\%) was used in this study. The thin film was fabricated by DC magnetron sputtering. The sputtering conditions were as follows: a base pressure of $5 \times 10^{-7}$ Torr, Ar pressure of 5 mTorr, and sputtering power of $50 \mathrm{~W}$. An in-plane magnetic field of 500 Oe was applied during sputtering to form an induced anisotropy. The thickness of the film was measured with a surface profiler. The $M-H$ hysteresis loops were measured using a vibrating sample magnetometer under in-plane magnetic fields at room temperature. The microstructure was examined using the X-ray diffraction (XRD) technique, and the composition was analysed using the inductively coupled plasma method. Elliptical cells, which were fabricated using a conventional photolithography technique, were used to form the shape anisotropy. The cells had a fixed short axis of $10 \mu \mathrm{m}$, but had three different long axes of 10,20 , and $50 \mu \mathrm{m}$, which allowed for three different aspect ratios $(r)$ of 1,2 , and 5, respectively. An array of cells on a $10 \mathrm{~mm} \times 10 \mathrm{~mm} \mathrm{Si}$ substrate, rather than a single cell, was used in this study, to obtain a large magnetic signal during the measurements. The cells were widely separated to avoid any cross-talk effects.

\section{Results and Discussion}

No crystalline peaks were observed from the XRD of the Co-Fe-Pd-B thin film, thereby indicating that its microstructure is comprised of an amorphous phase. Figure 2 shows the hysteresis loops of an unpatterned CoFe-Pd-B thin film along the easy and hard directions. A 


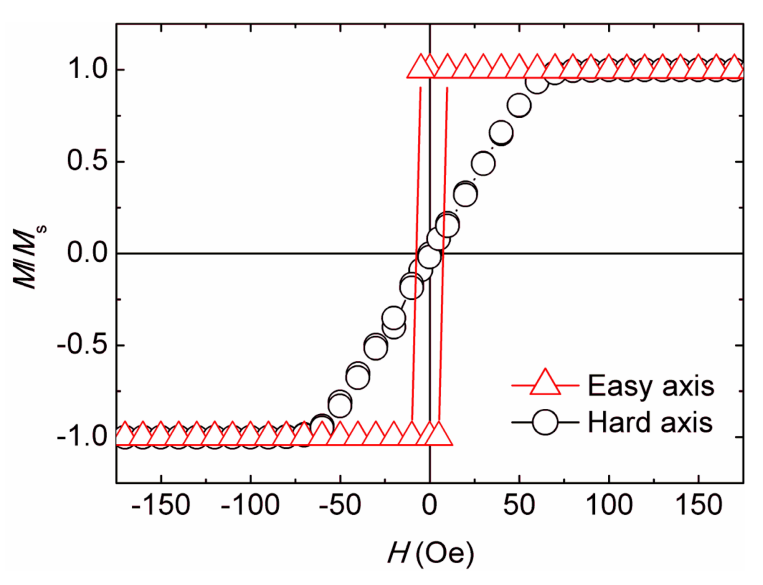

Fig. 2. (Color online) In-plane hysteresis loops along the easy (triangles) and hard (squares) directions of the unpatterned CoFe-Pd-B thin film used in this study.

clear difference exists between the two loops, thereby indicating the formation of a well-developed induced anisotropy. Two prominent features are: the absence of any hysteresis in the loop along the hard direction, which indicates a good anisotropy distribution, and the strength of the induced anisotropy (57 Oe), which is significantly larger than the values (usually below 20 Oe) observed in other amorphous-alloy thin films [13].

The shapes of the hysteresis loops of the patterned cells are very different from that of an unpatterned thin film. Figure 3(a) shows the hysteresis loops along the two principal directions for a circular cell with a diameter of $10 \mu \mathrm{m}$ and no shape anisotropy. The easy-axis loop of the unpatterned cell is completely squared with a remanence ratio of $100 \%$, but that of the patterned cell is inclined with nearly zero remanence. The hard-axis loop has a similar shape, but with a higher degree of loop inclination. This change in the shape of the loops as the thin film is patterned into a micron-sized cell is due to the demagnetizing fields, which cause a large change in the effective fields acting on the magnetic thin film. If these demagnetizing fields are properly corrected, then the hysteresis loops of the patterned cell could have an identical shape to those of the unpatterned thin film. This correction, however, appears to be very difficult in the present micronsized cell, in which the demagnetizing fields are quite nonuniform over the cell and the magnetic configuration deviates greatly from a single domain state. Even in this case, the difference in the saturation fields between the loops along the hard and easy directions should result from the induced anisotropy of the circular cell with no shape anisotropy. Indeed, this mechanism is confirmed with the results shown in Fig. 3(a), i.e., the difference in the saturation fields between the two loops being identical

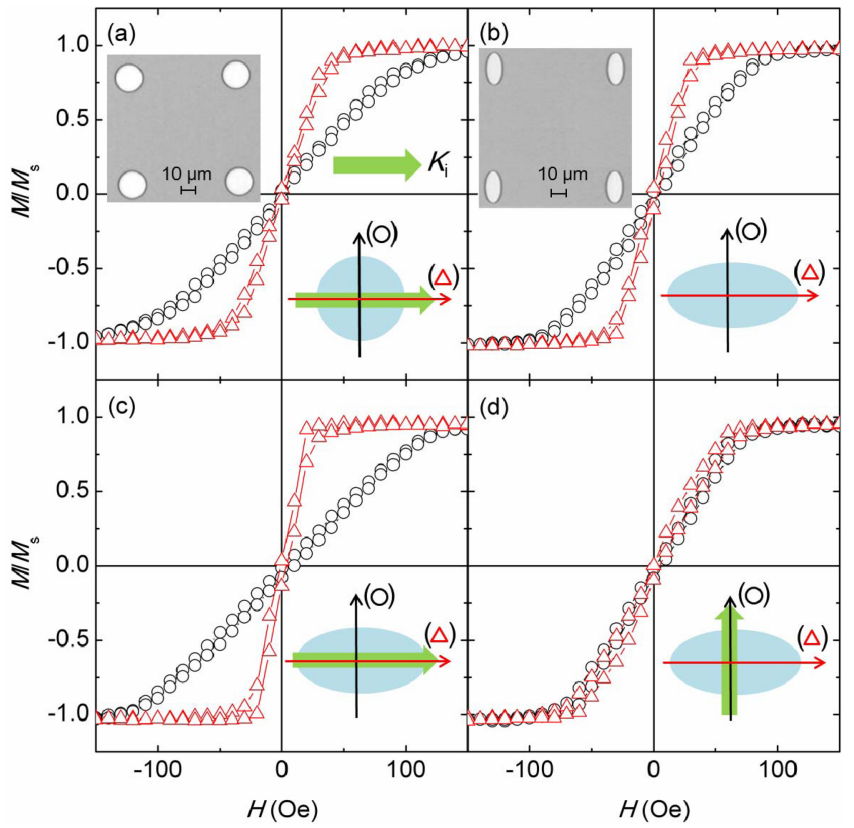

Fig. 3. (Color online) In-plane hysteresis loops along the two principal directions of (a) a circular cell with a diameter of 10 $\mu \mathrm{m}$ having only an induced anisotropy, (b) an elliptical cell of $10 \mu \mathrm{m}$ (short axis) $\times 20 \mu \mathrm{m}$ (long axis) having no induced anisotropy, (c) an elliptical cell of $10 \mu \mathrm{m}$ (short axis) $\times 20 \mu \mathrm{m}$ (long axis) having induced anisotropy, whose direction is parallel to the long axis of the cell, and (d) an elliptical cell of 10 $\mu \mathrm{m}$ (short axis) $\times 20 \mu \mathrm{m}$ (long axis) having induced anisotropy, whose direction is parallel to the short axis of the cell. Upper left insets in (a) and (b) show optical images of part of the arrays of circular and elliptical cells, respectively. Schematics in the lower right insets in all the figures show the directions of induced anisotropy (thick arrows) and $H$ (thin arrows).

to the strength of the induced anisotropy (57 Oe).

The magnitude of shape anisotropy can be similarly obtained from the difference in the saturation fields between the loops along the easy and hard directions, as shown in Fig. 3(b) for an elliptical cell of $10 \mu \mathrm{m}$ (short axis) $\times 20 \mu \mathrm{m}$ (long axis) with $r=2$. For this case, no induced anisotropy formed in the thin film during sputtering. The saturation field along the short (hard) axis is greater than that along the long (easy) axis by $51 \mathrm{Oe}$, which corresponds to the strength of the shape anisotropy of the elliptical cell. It is important to note that the shape anisotropy is of similar strength to the induced anisotropy. This strength of shape anisotropy was deliberately achieved by adjusting the cell dimensions, because the coupling effects are strong when mixed anisotropies are of similar strengths [14].

Samples with a single anisotropy (induced or shape) have been considered thus far. The change in the magnetic 
properties can similarly be examined when two anisotropies are coupled together. We considered two cases: the induced anisotropy is parallel to the shape anisotropy $(\beta=$ $0^{\circ}$ ), and two anisotropies cross at right angles $\left(\beta=90^{\circ}\right)$. The results for $\beta=0^{\circ}$ are shown in Fig. 3(c). The saturation field along the easy direction is reduced, but the saturation field along the hard direction is increased. Consequently, the difference in the saturation fields along the two principal directions increases, which agrees with the expected values. Quantitatively, a difference of 107 Oe was observed, which agrees well with the sum of the two original anisotropies (108 Oe). For the $\beta=90^{\circ}$ case, whose results are shown in Fig. 3(d), the difference in the saturation fields decreases to $6 \mathrm{Oe}$, which agrees well with the difference of the two original anisotropies (6 Oe). These results clearly indicate that for both cases, i.e., $\beta=0^{\circ}$ and $90^{\circ}$, no coupling occurs $(\eta=0)$ when the induced and shape anisotropies are mixed together.

Similar experiments were also performed using a $10 \mu \mathrm{m}$ (short axis) $\times 50 \mu \mathrm{m}$ (long axis) cell with $r=5$. The strength of shape anisotropy, which was determined using the same method used for the cell with $r=2$, was 98 Oe. In order to examine the anisotropy coupling, the difference in the saturation fields along the two principal directions was determined. It was found to be 145 Oe for $\beta=0^{\circ}$ and 35 Oe for $\beta=90^{\circ}$. These values are similar to the values of 155 Oe for $\beta=0^{\circ}$ and 41 Oe for $\beta=90^{\circ}$, which are expected if no anisotropy coupling is present, thereby further confirming that no anisotropy coupling occurs between the two uniaxial anisotropies. The slight differences emerge from extrinsic factors, such as the difficulty in forming a precise elliptical geometry and large change

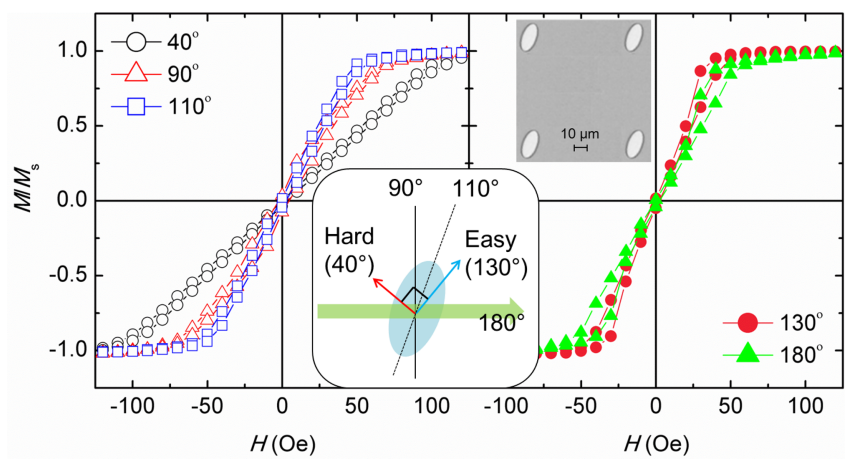

Fig. 4. (Color online) In-plane hysteresis loops of an elliptical cell of $10 \mu \mathrm{m}$ (short axis) $\times 20 \mu \mathrm{m}$ (long axis) having induced anisotropy, whose direction is located at $110^{\circ}$ with respect to the long axis. The loops are shown for some important angles of $H$. Upper inset shows an optical image of a part of arrays of elliptical cells and lower inset displays the schematic showing some important directions, including the directions of induced and shape anisotropies and easy and hard axes. in the magnetic properties as a function of the $H$ direction when the $r$ value of the cell is large.

Thus far, the coupling behaviour has been examined for the two cases of $\beta\left(0^{\circ}\right.$ and $\left.90^{\circ}\right)$, and the results indicate the absence of coupling $(\eta=0)$ between the two uniaxial anisotropies. In order to further confirm this coupling behaviour, a system with $\beta=110^{\circ}$ was examined. It consists of an elliptical cell with axes of $10 \mu \mathrm{m} \times 20 \mu \mathrm{m}$, with its long axis aligned at an angle of $110^{\circ}$ from the induced anisotropy. Hysteresis loops were measured over the entire range of angles at small steps to locate the easy and hard directions. Figure 4 shows the selected results for some of the angles. Careful analysis of the results indicates that there is only one easy axis at an angle of $130^{\circ}$, which is neither the induced nor shape anisotropy direction. The hard axis is located at $40^{\circ}$, which is exactly $90^{\circ}$ away from the easy axis. These results for $\beta=110^{\circ}$ indicate no anisotropy coupling $(\eta=0)$, which is identical to the behaviour observed for $\beta=0^{\circ}$ and $90^{\circ}$. For this case, however, the behaviour is not in strict agreement with the prediction from Eq. (1) because of the following two reasons. First, the difference in the saturation fields along the hard $\left(40^{\circ}\right)$ and easy $\left(110^{\circ}\right)$ directions, which is a measure of the anisotropy strength after coupling, should be smaller than the strength of the original anisotropies; however, the magnitude of the observed difference (57 Oe) is similar to those of the induced $(57 \mathrm{Oe})$ and shape anisotropies $(51 \mathrm{Oe})$. Second, the easy axis after coupling should be located at an angle closer to the induced anisotropy with the greater strength, but the observed easy axis is closer to the shape anisotropy. The reason for these discrepancies is not clear at this time and a further study is necessary to attain a fuller understanding of this coupling behaviour.

\section{Conclusions}

The anisotropy coupling in thin films with a mixture of induced and shape anisotropies was examined using an array of micron-sized cells with $r=1,2$, or 5. A 200-nmthick Co-Fe-Pd-B thin film with a large induced anisotropy of 57 Oe was used for the study. Three different angles, $0^{\circ}, 90^{\circ}$, and $110^{\circ}$, between the two anisotropies were considered. The results for the in-plane hysteresis loops, which were measured along many different directions, indicated no anisotropy coupling $(\eta=0)$ between the two uniaxial anisotropies. The agreement between the experimental and theoretical results, the latter obtained from Eq. (1), was observed to be good for $\beta=0^{\circ}$ and $90^{\circ}$, but it was not as strong for $\beta=110^{\circ}$, whose direction and strength of the resultant anisotropy after coupling differ 
from those predicted from Eq. (1).

\section{Acknowledgment}

This work was supported by the National Research Foundation of Korea (NRF) grant funded by the Korea government (MEST) (2011-0028163).

\section{References}

[1] D. Jiles, Introduction to Magnetism and Magnetic Materials, 2nd ed., Chapman and Hall, London (1998), Chaps. $12,13$.

[2] M. Takahashi, J. Appl. Phys. 33, 1101 (1962).

[3] S. Chikazumi, J. Phys. Soc. Jpn. 11, 551 (1956).

[4] D. Sander, Rep. Prog. Phys. 62, 809 (1999).

[5] E. P Wohlfath and D. G Tonge, Philos. Mag. 2, 1333 (1957).

[6] H. F. Du, H. L. Liu, Y. P. Fang, Q. Wu, T. Zou, X. Q.
Zhang, Y. Sun, and Z. H. Cheng, Appl. Phys. Lett. 96, 142511 (2010).

[7] Y. J. Nam and S. H. Lim, Thin Solid Films 519, 8256 (2011).

[8] N. A. Morley, M. R. J. Gibbs, E. Ahmad, I. G. Will, and Y. B. Xu, J. Phys.: Condens. Matter 17, 1201 (2005).

[9] H. Shima, K. Oikawa, A. Fujita, K. Fukamichi, K. Ishida, and A. Sakuma, Phys. Rev. B 70, 224408 (2004).

[10] A. Garcia-Arribas, E. Fernandez, A. V. Svalov, G. V. Kurlyandskaya, A. Barrainkua, D. Navas, and J. M. Barandiaran, Eur. Phys. J. B 86, 136 (2013).

[11] P. Zou and J. A. Bain, IEEE Trans. Magn. 36, 2536 (2000).

[12] X. Zhang, Q. Zhan, G. Dai, Y. Liu, Z. Zuo, H. Yang, B. Chen, and R.-W. Li, J. App. Phys. 113, 17 A901 (2013).

[13] G. Herzer, Acta Mater. 61, 718 (2013).

[14] Y. J. Nam and S. H. Lim, Appl. Phys. Exp. 5, 063002 (2012). 